\title{
Conditioned media from cell lines: A complementary model to clinical specimens for the discovery of disease- specific biomarkers
}

\author{
Paul Dowling and Martin Clynes \\ National Institute for Cellular Biotechnology, Dublin City University, Dublin, Ireland
}

In the strictest sense, the cell secretome (conditioned media) refers to the collection of proteins that contain a signal peptide and are processed via the endoplasmic reticulum and Golgi apparatus through the classical secretion pathway. More generally, the secretome also encompasses proteins shed from the cell surface and intracellular proteins released through non-classical secretion pathway or exosomes. These secreted proteins include numerous enzymes, growth factors, cytokines and hormones or other soluble mediators. They are fundamental in the processes of cell growth, differentiation, invasion and angiogenesis by regulating cell-to-cell and cell-to-extracellular matrix interactions. The main aim of this review is to provide a synopsis of findings from the analysis of the secretome taking diabetes, cancer and neurodegenerative diseases as examples. We will also discuss the preparation of conditioned media and on the main proteomic-based methodological approaches that have been developed for the study of secreted/shed proteins.

Received: August 23, 2010

Revised: September 8, 2010 Accepted: September 16, 2010

\section{Keywords:}

Biomarkers / Biomedicine / Cell culture / Conditioned media / Secretome

\section{Introduction}

Cell lines derived from tumours and tissues are widely used in laboratory-based research because they reflect some of the genotypic and phenotypic characteristics of primary tissues under investigation. Cell lines have been extensively used, for example, in screening studies involving drug sensitivity/ resistance of novel agents, to create tumours in the mouse models and to investigate characteristics such as invasion and cell division $[1,2]$.

Correspondence: Dr. Paul Dowling, National Institute for Cellular Biotechnology, Dublin City University, Glasnevin, Dublin 9, Ireland

E-mail: paul.dowling@dcu.ie

Fax: +353-1-7005484

Abbreviations: GDF15, growth/differentiation factor 15; GSIS, glucose-stimulated insulin secretion; HFC, hollow fiber culture; IFN, interferon; IHC, immunohistochemistry; LPS, lipopolysaccharide; MRM, multiple reaction monitoring; NPC, nasopharyngeal carcinoma; PTX3, pentraxin 3; TFF3, trefoil factor 3; TGFßlp, transforming growth factor- $\beta$-induced protein; VGF, nerve growth factor inducible
Over the last number of years, many research groups have analysed conditioned media under well-defined experimental conditions to aid in their biomarker discovery investigations (Table 1). Proteins released into conditioned media by cultured cells are a rich source of material for biomarker discovery experiments [3]. Certain proteins secreted from cells that enter the circulatory system can be exploited as molecules for screening for the presence of disease or monitoring therapeutic effectiveness. This technique provides a useful and less invasive alternative to direct clinical specimen analysis for the discovery of novel biomarkers. By using a cellculture model system in which the cells were grown in serum-free media, the identification of candidate biomarkers through the use of proteomic analysis is enhanced due to a lower dynamic range, spanning five or six orders of magnitude [4] and a smaller diversity of proteins. Large data sets of secreted or shed proteins have emerged as candidate biomarkers from studies of cell populations, and many of these have been initially validated in clinical

Colour Online: See the article online to view Fig. 2 in colour. 
Table 1. Cell lines that have been used by researchers during proteomic investigations of the respective secretomes

\begin{tabular}{lll}
\hline Disease type & Cell lines & Ref. \\
\hline Diabetes & 3 TT-L1 & {$[48]$} \\
& MIN-6 & {$[49]$} \\
INS-1 & {$[50]$} \\
PAncer & NPC-1 & {$[50]$} \\
& CL $1-0$ & {$[56]$} \\
& CL1-5 & {$[57]$} \\
MCF10AT & {$[57]$} \\
& MCF10 DCIS.com & {$[58]$} \\
& MCF 10CA cl. D & {$[58]$} \\
& SW480 & {$[58]$} \\
& SW620 & {$[59]$} \\
& PC3 & {$[59]$} \\
& LNCaP & {$[60]$} \\
& 22Rv1 & {$[60]$} \\
& AsPC1 & {$[60]$} \\
& MiaPaCa2 & {$[37]$} \\
& PANC1 & {$[37]$} \\
& PT45 & {$[37]$} \\
BV-2 & {$[37]$} \\
& B35 & {$[68]$} \\
& C6 & {$[68]$} \\
SH-SY5Y & {$[69]$} \\
& & {$[70]$} \\
\hline
\end{tabular}

Included is the disease type, cell line and associated reference.

samples achieving high levels of sensitivity and specificity [5-8].

Proteins identified in media conditioned by cell lines will exit the cells through a number of different pathways. During the classical secretory pathway, proteins containing an ER (endoplasmic reticulum) signal sequence, generally at the N-terminus, are secreted in an ER/Golgidependent manner [9]. During secretion through the non-classical pathway, no signal sequences are present and proteins are released by a variety of mechanisms, including shedding of plasma membrane microvesicles, direct efflux through plasma membrane transporters and exocytosis of secretory lysosomes/exosomes [10]. Exosomes are small $(40-90 \mathrm{~nm})$ membrane vesicles of endocytic origins that harbour components of the secretome including membrane and cytoplasmic proteins [11]. It is also likely that alternative pathways or mechanisms are present for proteins to exit cells that have yet to be discovered (Fig. 1).

Biofluids such as plasma and serum are the ideal samples to investigate from a biomarker discovery perspective. They have a very high protein concentration and act as a reservoir to concentrate signals from all tissue types present in the body. However, only 22 proteins account for $99 \%$ of their protein content and include albumin, haptoglobin, transferrin, immunoglobulins and complement factors. Albumin, for example, exists at concentrations in excess of a billion times more concentrated than many

\section{Signal Peptides}

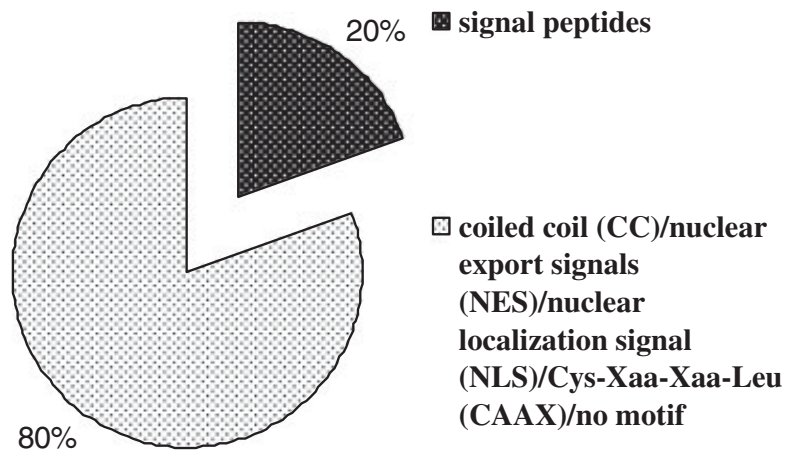

Figure 1. Analysis of conditioned media from colon cancer cell lines. In-solution digestion and MS analysis of conditioned media from four colon cell lines - HCT116, HT-29, SW480 and WiDr demonstrated that 92 proteins were common to all four. Further analysis of these 92 proteins showed that 18 of them had a signalling peptide for processing through the classical secretory pathway. As shown in the pie chart, the majority of common proteins exited the cells by alternative pathways.

cytokines and it is very likely that tumour-specific biomarkers will be present at concentrations that are much lower [12]. Unfortunately, plasma and serum proteins are present across a dynamic range of concentration that is likely to span more than 12 orders of magnitude, making it extremely difficult to analyse their respective proteomes comprehensively.

Another considerable challenge for biomarker discovery investigations in biofluids is the susceptibility of lowabundance biomarkers to rapid degradation by endogenous and exogenous proteinases upon release from the tumour and also during sample processing [13]. Many low-abundant proteins are sequestered by high-abundant circulatory proteins which provide a certain amount of protection from degradation but also add to the complexity of analysing these candidate biomarkers. Thus, biofluid-based approaches for biomarker discovery still represent a significant challenge and require highly specialized technologies. Therefore, analysis of conditioned media has many advantages over direct analysis of clinical specimens. These advantages include the non-invasive method of sample collection and the fact that a large number of cell lines representing various stages/histotypes are readily available [14]. The identification/quantification of these secreted or shed proteins is relatively straightforward without the problems that are associated with high-abundance proteins or the dynamic range associated with many biofluids $[15,16]$.

Advances in mass spectrometry (MS), label/label-free technologies, LC systems and bioinformatic approaches have had a tremendous influence on proteomics [17]. These advances combined with the analysis of conditioned media will undoubtedly facilitate the identification of candidate 
biomarkers, initial validation in clinical samples and detection of the most promising molecules in terms of medical utility.

\section{Sample preparation}

Serum-supplemented media can present analytical challenges due to the masking effects of highly abundant serum proteins, such as bovine serum albumin (BSA), for example. Analysis of total lysates from cultured cells frequently results in the identification of serum proteins and this problem is amplified when trying to investigate proteins secreted or shed into the media. Our group has previously looked at culturing cells in different concentrations of serum, ranging from 0 to $10 \%$ with subsequent analysis of secreted proteins. Even at $0.5 \%$ serum, the masking affect of the high-abundant proteins was sufficient to make the analysis of secreted proteins extremely difficult. Many groups have reported on novel washing and culturing technique for cells that considerably reduce the concentration of high-abundant supplemented serum proteins in the secretome of these cells. Pellitteri-Hahn et al. described a method consisting of rinsing twice with $10 \mathrm{~mL}$ of Dulbecco's phosphate-buffered saline with calcium and magnesium (DPBS) and once with $10 \mathrm{~mL}$ of serum-free media that showed a significant reduction in BSA peptides identified by MS when compared with the no-rinse control cells [18]. The percentage of contaminant BSA scans identified by MS in the stringent wash (average, 13.2\%) was much lower than either the moderate or the no-rinse treatments (average, 35.2 and $45.2 \%$ ).

In the majority of publications, cells are washed with PBS or serum-free media or a combination of both. The Srisomsap et al. protocol for collection of conditioned media consisted of washing the cells with serum-free medium two times before incubation in serum-free medium for $24 \mathrm{~h}$ [19]. The Higa et al. procedure involved extensive washing of the cells with PBS and incubated for $16 \mathrm{~h}$ with serum-free Dulbecco's-modified Eagle medium [20]. It is clear from the literature that each group has optimized their own specific approach for washing cells prior to incubation with serum-free media. Certainly, there exists a fine line between not washing the cells sufficiently, risking the masking effects of BSA and other serum components and washing the cells too stringently, a process that can damage or kill the cells and lead to a release of contaminating cytoplasmic proteins.

Other variables to consider when preparing conditioned media samples for proteomic analysis are incubation time and cell confluency. Both of these factors can have a significant effect of the quality of the samples and certainly warrant careful optimization. Some groups opt to monitor the levels of contaminating cytosolic proteins in the media as a measure of how conditions are affecting the quality of the secreted protein purity in the conditioned media. For example, Mbeunkui et al. demonstrated that the level of the major cytosolic proteins $\beta$-actin and $\beta$-tubulin increased significantly in the conditioned medium after $30 \mathrm{~h}$ or when the cell confluency was above $70 \%$ due to cell autolysis. Subsequent monitoring of $\beta$-actin and $\beta$-tubulin resulted in an incubation time of $24 \mathrm{~h}$ and $60-70 \%$ cell confluency being considered as the optimal incubation time and amount of cells respectively, during conditioned medium analysis [21]. Weng et al. monitored the relative distribution of $\beta$-tubulin, an abundant cytosolic protein, in the conditioned media and in the extracts of residual cells attached on the culture dishes by Western blot analysis. Their data show that $\beta$-tubulin was detected in the total cell extracts but not in the conditioned media, suggesting that the release of proteins into the conditioned media was not caused by cell lysis and the incubation in serum-free medium for $24 \mathrm{~h}$ was well controlled [22]. Therefore, careful optimization of serum-free conditioned media collection is an important step, however, with any protocol there is still always the risk that contaminating abundant serum proteins and cytosolic proteins will be identified in subsequent investigations.

There is also a concern that culturing cells in serum-free media for periods ranging from a few hours and many days could impact on the phenotypic characteristics of the cells compared with standard conditions and therefore impinge on the nature of protein secreted or shed into the media. Zwickl et al. demonstrated a method involving culturing HepG2 cells using standard conditions using methionine and cysteine-free William's E medium (ICN) in the presence of ${ }^{35} \mathrm{~S}$-labelled methionine and cysteine. Metabolic labelling of cells allows the detection of proteins by autoradiography, when only proteins synthesized by living cells during the metabolic labelling period will be visualized [23]. More recently, Gundacker et al. also used ${ }^{35}$ S-labelled methionine and cysteine to identify the secreted proteins in the supernatant of cultured dendritic cells [24].

Colzani et al. recently described the use of an LC-MS/MSbased strategy to study serum-containing conditioned media which combines metabolic labelling with protein equalization using Proteominer beads [25]. An aggressive breast cancer cell line (MDA-MB-231) grown in a standard medium with $10 \%$ FCS was labelled with an excess of deuterated valine (D8-Val). The obtained conditioned media was equalized and after protein identification, the presence of the isotope label was used to discriminate true cellular proteins from homologous bovine ones. Quantitative analysis was performed by comparing the secretome of MDA-MB-231 with a less aggressive breast cancer cell line, MCF-7. Relative quantification of validated cellular proteins was done using spectral counting and confirmed for three proteins LG3BP, IBP2 and FLNA using selected reaction monitoring (SRM).

Methods for extracting proteins from conditioned media include precipitation, ultrafiltration and lyophilization [26]. 
Like all methods for purifying protein, none of these are $100 \%$ efficient and some are more suitable to certain types of proteins if they are of primary interest. Trichloroacetic acid/acetone precipitation is an effective approach but has an inherent disadvantage that there is always a certain amount of protein loss and subsequent re-solubilization of proteins can prove troublesome. Ultrafiltration is a popular choice when concentrating proteins in conditioned media as this method is relatively straightforward, cost effective and very reproducible [27].

\section{Proteomic approaches for conditioned media examination}

Applying advanced proteomic-based separation and identification approaches to the investigation of media conditioned by cell lines, researchers have dramatically increased the number of candidate biomarkers identified in such samples. Different proteomic platforms such as two-2-D PAGE [28], 2-D DIGE [29], SELDI-TOF-MS [30], protein arrays [31], iTRAQ [32] and MudPIT (multidimensional protein identification technology) [33] have been used to examine various biological samples including conditioned medium to discover, validate and characterize disease-associated proteins.

Stable isotope labelling technology, by both metabolic and chemical methods, has been a very successful approach used to profile wide-ranging proteomes in combination with MS [34]. Perhaps, the most suitable labelling technology when examining the secretomes of cell lines from disease compared with normal populations or with differing levels of invasive potential, for example, is stable isotope labelling with amino acids in cell culture (SILAC). Cells are grown in identical conditions, with one cell line incorporating heavy isotopic amino acids, usually Arginine (U-13C6, 15N4) and Lys (U-13C6) thereby taking advantage of the normal metabolic machinery of the cell to label the proteins. The light (natural isotope) and heavy amino acids are chemically identical and thus co-elute upon LC-based separation. But, since the peptides are isotopically distinct, the light- and heavy-labelled peptides are clearly distinguishable by MS, allowing for comparable abundance levels to be established [7, 35].

Label-free quantitation methods are gaining in popularity. The technique does not use any labelling of proteins, but instead directly compares signal intensities between different LC/MS runs [36, 37]. A fundamental component of label-free quantitation is MS, which has become an essential analytical technology in modern biological and medical research. MS-based platforms are able to successfully interpret the increasing number of proteins from biological samples, such as conditioned media and together with advanced bioinformatics programmes have the ability to become a powerful tool for biomarker discovery and validation.
Validation of candidate biomarkers discovered through screening of conditioned media in clinical samples is the ultimate step when analysing the secretomes of various cell lines, providing information on the clinical utility and accuracy of the candidate biomarkers. ELISA, Western blot analysis, immunohistochemistry (IHC) and multiple reaction monitoring (MRM) are methods that are commonly used to evaluate conditioned media proteins in clinical samples. ELISA, Western blotting and IHC relay on the availability of quality antibodies to produce consistent valid results. ELISAs are routinely used in the clinic and hence would be the ideal final test assay for any new biomarkers. IHC is a valuable technique utilized to localize/ visualize protein expression in a mounted tissue section using specific antibodies. IHC can also be scored in the form of a semi-quantitative result, giving a general indication of the abundance levels for the specific protein being probed [38].

Immunoassay-based validation approaches, such as ELISA tests, are relatively easy to perform, but they are expensive and may take a long time to develop, especially if antibodies for the candidate biomarkers are not immediately available. To this end, MRM, a highly specific and sensitive MS technique has been developed that can selectively quantify proteins within complex mixtures such as serum or plasma samples [39]. An MRM experiment is accomplished by specifying the parent mass of a peptide for MS/MS fragmentation in Quadrupole 1 (Q1), and then specifically monitoring for a single fragment ion from the parent in Quadrupole $3(\mathrm{Q} 3)$ using a triple quadrupole instrument (QQQ). MRM delivers a unique signal (Q1-Q3 transition) that can be monitored and quantified, using synthetic stable isotope labeled peptide as standards, in the middle of a very complex environment. The sensitivity of MRM is also considerably increased when used in combination with Stable Isotope Standards and Capture by Anti-Peptide Antibodies (SISCAPA), a method for enriching target peptides using anti-peptide antibodies [40].

\section{Diabetes secretome analysis}

Populations worldwide are currently experiencing an epidemic of obesity with many associated health problems, including type 2 diabetes, formerly classified as non-insulindependent diabetes mellitus (NIDDM) or adult-onset diabetes. Type 2 diabetes is a complex and heterogeneous disease caused by both environmental and/or genetic factors and affects more than 150 million people worldwide, a figure that is increasing at an alarming rate [41]. Type 2 diabetes is the principal cause of blindness, kidney failure and lower limb amputation, as well as one of the major risk factors for heart attack and stroke [42-44].

Adipose tissue has been shown to be a primary component of type 2 diabetes progression by establishing and maintaining a chronic state of low-level inflammation. This 
role is mainly determined by the adipose secretome, in particular because of the huge diversity of soluble signaling factors that are secreted from adipose tissue $[45,46]$. Therefore, a greater understanding of the adipose secretome using proteomic-based platforms may help combat this rapidly expanding disease.

Zvonic et al. [47] examined the secretome of primary cultures of human subcutaneous adipose-derived stem cells as an in vitro model of adipogenesis. Conditioned media obtained from four individual female donors after culture in uninduced or adipogenic-induced conditions were compared by 2-D gel electrophoresis and MS/MS. Of particular interest was the presence of multiple serine protease inhibitors (serpins), a result that suggests this protein family could play a significant role with respect to the etiology of obesity and type 2 diabetes. Zhou et al. recently employed cleavable isotope-coded affinity tag (cICAT) and label-free quantitation approaches to identify and quantify secretory factors that are differentially secreted by 3T3-L1 adipocytes with or without insulin treatment. In total, 317 proteins predicted or annotated as secretory proteins were identified using this approach [48]. A total of 77 reported adipokines were quantified in this study, such as adiponectin, cathepsin D, cystatin C, resistin and transferrin. Overall, these results suggest that insulin stimulation might up-regulate the glycosidases secreted from adipocytes, and these secretory glycosidases might modify their glycan targets in other location through systematic circulation.

Our group has previously worked with media conditioned by pancreatic $\beta$-cell models. Low-passage MIN-6 (mouse insulinoma) cells respond to changes in glucose concentrations, producing an approximately 5.5-fold glucose-stimulated insulin secretion (GSIS) in response to $26.7 \mathrm{mmol} / \mathrm{L}$. After continuous culture or high passage, this GSIS was no longer present and thus represents an excellent model system for investigating $\beta$-cell dysfunction (Fig. 2). Proteomic analysis of the conditioned media revealed that secretogranin II, secretogranin III and transthyretin, proteins associated with secretory granule biogenesis and were found to have substantially increased levels in non-responsive high-passage MIN-6 $\beta$ cells and may be clinically useful for assessing $\beta$-cell function and monitoring the effects of specific therapeutics [49].

Song et al. analysed the effects on the proteome of pancreatic islet $\beta$ cells with and without pancreatic cancer cell-conditioned medium stimulation [50]. In this study, it was observed that the pancreatic cancer cell-line PANC1-conditioned media decreased insulin secretion from rat islet $\beta$-cell INS-1. A total of ten up-regulated proteins and five down-regulated proteins were identified in pancreatic cancerconditioned media-treated $\beta$-islet cells compared with negative controls. Factors released by the pancreatic cancer cell line into the conditioned media affected the expression levels of HSP60, Prp19, peripherin, HMOX1 and GRP78 expression in islet $\beta$ cells, establishing a link between these proteins and pancreatic cancer-associated diabetes.

\section{Cancer secretome analysis}

According to GLOBOCAN, an estimated 12.7 million new cancer cases and 7.6 million cancer deaths occurred in 2008 (http://globocan.iarc.fr/). The most frequent types of cancer worldwide among men are lung, stomach, liver, colorectal, oesophagus and prostate. Among women, breast, lung, stomach, colorectal and cervical are the most prevalent [51]. Recent developments in the identification of biomarkers may help in the early detection of cancer, aid prognosis, predict therapeutic response and also increase our general understanding of the underlying pathobiochemical mechanisms of specific diseases. Generally, biomarkers are produced by either the tumour itself or other tissues in response to the presence of cancer, with panels of biomarkers likely to contain both tumour-specific and tumour nonspecific proteins [52, 53].

A large number of studies have focused on analysing the conditioned media from cancer cells, specifically trying to identify candidate biomarkers to potentially validate in clinical samples. Many groups examine the secretome from cancer cells compared with normal cells, with a particular emphasis on detecting signatures that are unique to the cancer cells and therefore may represent possible early detection biomarkers. Recently, Matsumoto et al. analysed peptides which were secreted into conditioned medium by LCN1, a large cell neuroendocrine carcinoma cell line using MALDI-TOF MS [54]. Two fragments of nerve growth factor inducible (VGF) were identified in the conditioned media and were subsequently analysed using RT-PCR analysis of lung cancer cell lines. This data demonstrated that VGF mRNA was expressed only in neuroendocrine carcinomaderived cells, a result that demonstrates the potential of VGF as a novel serological diagnostic marker of pulmonary neuroendocrine tumours.

Nasopharyngeal carcinoma (NPC) is a rare malignancy arising from the epithelium of the nasopharynx. This malignancy is uncommon in most parts of the world, but is one of the most common cancers in Southeast Asia [55]. The number of candidate biomarkers associated with NPC is relatively small but recently a publication by Chang et al. describes the identification of chloride intracellular channel 1 (CLIC1) together with 65 additional proteins found in the conditioned media from NPC-TW04 cells [56]. Using SignalP 3.0, SecretomeP 2.0 and TMHMM, to predict classical/non-classical pathway secretion, it was shown that 47 out of the 66 identified proteins could be secreted from NPC cells via the various mechanisms. In particular, the plasma levels of CLIC1 in NPC patients $(N=70)$ were significantly higher than those in the healthy controls $(N=74)$ $($ mean $\pm \mathrm{SD}, \quad 16.38 \pm 26.53$ versus $2.39 \pm 5.32 \mu \mathrm{g} / \mathrm{mL}$; $p=0.00005)$ when analysed using ELISA, resulting in a sensitivity of $63 \%$ and a specificity of $77 \%$.

Some groups compared conditioned media from cells with invasive and non-invasive phenotypes, other focus on identifying secreted proteins associated with drug resistance 


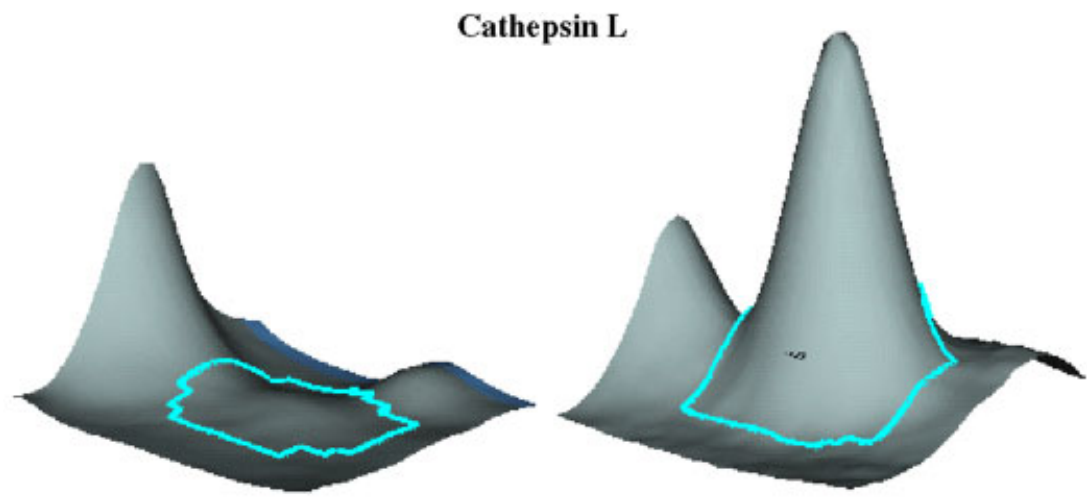

Figure 2. Analysis of conditioned media from low passage compared with high passage MIN-6 cells. Low-passage MIN-6 (mouse insulinoma) cells respond to changes in glucose concentrations, producing an approximately 5.5 -fold GSIS in response to $26.7 \mathrm{mmol} / \mathrm{L}$. After continuous culture or high passage, this GSIS was no longer present and thus represents an excellent model

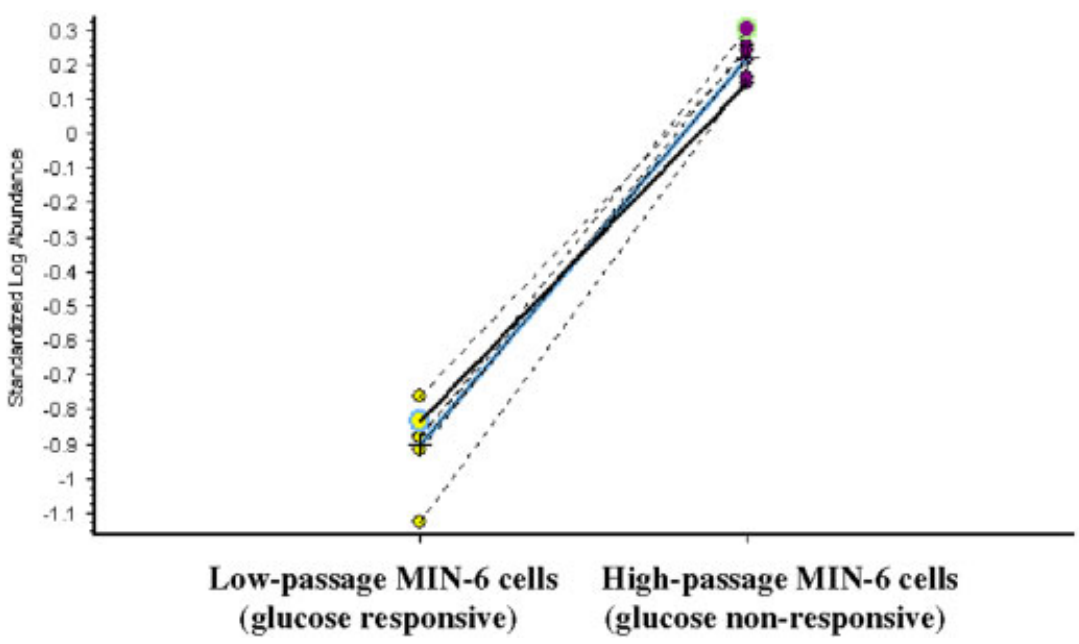
system for investigating $\beta$-cell dysfunction. Cathepsin L was found to be 13-fold increased in the conditioned media from low passage compared with high passage MIN-6 cells using 2-D DIGE analysis (see graph view). Cathepsin L (CTSL) is a ubiquitously expressed major representative of the papain-like family of cysteine proteinases and in this investigation represents a candidate biomarker for further clinical validation as an indicator of $\beta$-cell dysfunction. CyDye DIGE fluor minimal dyes that consist of three different CyDyes (Cy2, Сy3 and Cy5) were used in this analysis. Cy3-labelled lowpassage MIN-6 cells (glucose responsive) and Cy5-labelled high-passage MIN-6 cells (glucose non-responsive) are shown in 3-D view.

or metastasis. For example, Wang et al. used a combination of results from screening the conditioned media of two NSCLC cell lines, $\mathrm{CL}_{1-0}$ and $\mathrm{CL}_{1-5}$ with pleural effusion proteomic data to identify 22 protein of interest [57]. Retinoblastoma-associated binding protein 46 (RbAp46) was selected for further analysis and was found to be significantly higher in lung cancer patients compared with healthy controls $(34.0 \pm 15.7$ versus $22.3 \pm 16.2 \mathrm{ng} / \mathrm{mL}, p<0.001)$ and positively correlated with distal metastasis of NSCLC. Mbeunkui et al. analysed conditioned media from a series of isogenic breast cancer cell lines varying in aggressiveness by MS: non-tumourigenic MCF10A, premalignant/tumourigenic MCF10AT, tumourigenic/locally invasive MCF10 DCIS.com and tumourigenic/metastatic MCF 10CA cl. D [58]. Using LC-MS/MS, $\alpha$-1-antichymotrypsin and galectin3-binding protein among others were found secreted at high levels from MCF10 DCIS.com and MCF10CA cl. D, yet undetected in the MCF10A and MCF10AT cell lines. These results demonstrate that the analysis of an isogenic series of cell lines is potentially useful for identifying prospective markers for the early detection and aggressiveness/ progression of cancer.

Similarly, Xue et al. examined the secretome from a primary cell line SW480 and its lymph node metastatic cell line SW620 from the same colorectal cancer patient [59]. A total of 145 differential proteins between SW480 and SW620 ( $>1.5$-fold change) were identified. Among them, trefoil factor 3 (TFF3) and growth/differentiation factor 15 (GDF15), two up-regulated proteins in SW620, were further analysed in a large cohort of clinical tissue and serum samples. For discriminating CRC from healthy controls, the AUC was determined to be 0.730 ( $95 \%$ confidence interval, 0.670-0.791) for TFF3 and 0.897 (95\% confidence interval, 0.856-0.938) for GDF15. It was also observed that the high TFF3 or GDF15 serum levels were significantly associated with a higher histological grade, more advanced TNM stage and the presence of distant metastases.

Sardana et al. recently examined conditioned media from three human prostate cancer cell lines of differing origin (PC3 (bone metastasis), LNCaP (lymph node metastasis) and 22Rv1 (localized to prostate)) to identify secreted proteins that could serve as novel prostate cancer biomarkers [60]. Based on criteria including tissue specificity, biological function, disease association based on literature searches and comparison of protein overlap with the proteome of seminal plasma and serum, four novel candidates, follistatin, chemokine (C-X-C motif) ligand 16, pentraxin 3 (PTX3) and spondin 2 were validated in the serum of patients with and without prostate cancer. A significant difference (Kruskal-Wallis test) in sera of 
patients with or without $\mathrm{CaP}$ in all four candidates by ELISA was observed. In addition, the correlation between PSA levels and candidate levels in serum of patients with $\mathrm{CaP}$ was significant and positive by Spearman analysis.

Analysis of conditioned media from cancer cells not only allows researchers to identify candidate biomarkers for the detection of cancer, but could also offer in some instances a realistic opportunity to identify possible therapeutic targets [61]. It is possible that there is some crossover between biomarkers of detection/monitoring and possible drug targets, and hence it comes as no surprise that some of the conditioned media-identified candidate biomarkers are also mentioned in terms of therapeutic development by many groups. An example of this is the publication by Schiarea et al. [37]. The secretomes of four human pancreatic ductal adenocarcinoma (PDAC) cell lines, AsPC1, MiaPaCa2, PANC1 and PT45 were compared with a normal counterpart, the immortalized epithelial cell line derived from normal human pancreatic ducts, HPDE6. This group found proteins over-expressed in all of these PDAC cell lines including CD9, vimentin, PLOD3, SH3L3, PCBP1 and SFRS1. This group of proteins was therefore considered especially interesting as potential therapeutic targets or biomarkers for pancreatic cancer. Interestingly, other groups have proposed vimentin as a therapeutic target in cancer, evidence that supports the identification of potential targets through analysis of media conditioned by cell lines [62].

\section{Neurodegenerative disease secretome analysis}

Neurodegenerative diseases, such as Huntington's, Parkinson's, Alzheimer's, Machado Joseph disease, multiple sclerosis and Amyotrophic lateral sclerosis (ALS) represent a subgroup of human diseases with certain features in common, including being predominantly of adult onset. Disease pathogenesis usually involves the progressive loss of specific neuronal population's characteristic of each disease type. Millions of people worldwide suffer from neurodegenerative disorders, illnesses that normally manifest later in life. Alzheimer disease, the most prevalent of these disorders, affects 15 million people worldwide today, with the number of affected individuals in the United States and Europe expected to triple by the year 2050 to 13.2 million and 16.2 million, respectively [63, 64]. Chronic microglial activation is an important central component of neurodegenerative diseases, with release of proinflammatory and neurotoxic factors such as tumour necrosis factor- $\alpha$ (TNF- $\alpha$ ), interleukin-1 $\beta$ (IL-1 $\beta)$, free radicals such as nitric oxide (NO) and superoxide [65]. These chronic neuroinflammatory and neurotoxic components are likely to contribute to neuronal dysfunction and disease progression. Given that central nervous system (CNS) tissue has very limited, if any, regenerative capacity, it is of utmost impor- tance to limit the damage caused by neuronal death. Microglia, a pivotal cellular player in neuroinflammation has therefore been the subject of much research, including examining the secretome, in order to generate a greater understanding of the progression of this disease group [66-68].

To characterize secreted proteins of activated microglia, conditioned media obtained from BV-2 mouse microglia cells were analysed by 2-D gel electrophoresis or LC coupled with tandem MS by Kim et al. [69]. BV-2 cells were either left untreated or treated with lipopolysaccharide (LPS)/interferon (IFN), to maximally activate microglia, for 4-18h under serum-free condition. One of the proteins whose relative abundance was increased in the conditioned media of activated microglia was cathepsin D. Further analysis demonstrated that knockdown of cathepsin D expression in microglia using short hairpin RNA diminished the neurotoxicity in a co-culture system of BV-2 microglia cells and B35 neuroblastoma, used as a model of microglia/neuron coculture. The author proposed from this body of study that cathepsin D precursor protein can be a potential biomarker or drug target for the diagnosis and treatment of neurodegenerative diseases that are associated with excessive microglial activation and subsequent neurotoxic inflammation.

Jeon et al. recently screened the secretome of mixed glial cells stimulated with LPS and IFN- $\gamma$ for $24 \mathrm{~h}$ using LC-MS/ MS [70]. Among the proteins identified by the mixed glia secretome analysis, PTX3 secretion was most highly induced by LPS/IFN- $\gamma$ stimulation. The effect of PTX3 protein on phagocytic activity of microglia was subsequently investigated using zymosan particles with apoptotic cells and it was observed that addition of PTX3 protein enhanced the engulfment of zymosan particles by microglia. It was concluded that PTX3 may serve as potential biomarkers that help diagnose inflammatory brain disorders and predict the drug responses.

Hur et al. examined ischemia-induced damage to the CNS by analysing the effects of microglia (MCM)- or astrocyte (ACM)- conditioned media obtained by chemical ischemia on the neuronal injury in SH-SY5Y cells [71]. This investigation showed that chemical ischemia induced the expression and activation of microglial cytochrome $c$, inflammatory cytokines, reactive oxygen species (ROS) and NADPH oxidase in BV2 cells, resulting in neuronal cell death in SH-SY5Y cells. Therefore, a link between the generation of ROS and the interactions of astrocytes, microglia and neurons during ischemic brain injury was established.

\section{Limitations of conditioned media- based biomarker discovery}

Analysis of conditioned media has proven to be a very successful strategy for the identification of candidate biomarkers for further validation in clinical samples. 
However, this approach does suffer from some obvious limitations. Cell culture is an in vitro system and at a molecular level will be different from the in vivo situation. Ultimately, this difference will be reflected in the secretome, making a direct correlation between conditioned media and biofluid expressed proteins difficult.

Most human tumours, for example, are a heterogeneous mixture of different cells with the interaction between different cell types, resulting in a complex regulation of cell growth, differentiation and invasion [72]. Therefore, no single cell line can mimic this heterogeneity that is responsible for many of the characteristics associated with specific types of cancer. To mimic this, many groups choose to analyse a panel of cell lines for a particular cancer type to encapsulate the molecular diversity and subsequent range of proteins secreted or shed into the conditioned media $[19,22]$.

The interaction between the host and microenvironment influencing tumour development and progression is absent in 2-D culture systems together with associated morphological characteristics. To overcome some of these limitations, some researchers have developed more advanced systems, including the use of co- and three-dimensional (3-D) cultures. Analysis of the proteins secreted or shed into the conditioned media from a co-culture system may reflect better the interactions between the tumour cells and the immediate microenvironment in vivo. Co-culture studies have found that the survival and proliferation of cancer cells are intimately linked to the soluble factors in the microenvironment, such as epidermal growth factor (EGF) that contribute to tumour cell proliferation, morphology, angiogenesis and invasion. An example of the importance of this interaction and the identification of candidate biomarkers was a recent publication by Ween et al. [73]. This article investigates the ovarian cancer-peritoneal interaction by means of in vitro co-culture experiments with ovarian cancer (OVCAR-5 and SKOV-3) and peritoneal (LP-9) cells. One of the proteins differentially expressed in the coculture secretome was identified by MALDI-TOF/TOF MS as the extracellular matrix protein transforming growth factor$\beta$-induced protein (TGF $\beta$ Ip). In functional in vitro experiments, treatment with recombinant TGF 3 Ip significantly increased the motility and invasiveness of OVCAR-5 and SKOV-3 cells concluding that TGF $\beta$ Ip expressed by peritoneal cells increases the metastatic potential of ovarian cancer cells.

Zhong et al. postulated that stromal cells in the tumour microenvironment alter the tumour cell secretome, including those proteins required for tumour growth and dissemination [5]. To test this hypothesis, they developed an in vitro model based on coculturing a murine K-ras mutant lung adenocarcinoma cell line (LKR-13) with a murine lung stromal cell (macrophage, endothelial cell or fibroblast). They found that this co-culture system enhanced stromal cell migration, induced endothelial tube formation, increased LKR-13 cell proliferation and regulated the secretion of proteins involved in angiogenesis, inflammation, cell proliferation and epithelial-to-mesenchymal transition.

3-D culture systems are frequently used to examine the secretome of cell lines. 3-D cultures may exhibit cellular behaviours and morphologies similar to those seen in vivo when compared with 2-D cultures. An example of this in the literature is the development of a strategy for cell secretome analysis using a hollow fiber culture (HFC) system combined with LC-MS [56, 74, 75]. The HFC system provides high-density 3-D cell cultures that mimic solid tumours in vivo, and in addition, collects cell secretome with improved efficiency. The HFC system also greatly reduces cell lysis, which alleviates the contamination from intracellular proteins.

Cell line cross-contamination can be a problem for scientists working with cultured cells. Studies suggest that anywhere from 15 to $20 \%$ of the time, cells used in experiments have been misidentified or contaminated with another cell line [76, 77]. Many of the problems relate to HeLa cells, the first human cancer cell line to be established. This is an important factor for researchers to consider, and therefore careful quality control criteria must be adhered to before setting out on conditioned media-based investigations.

Disease-affected cells can induce the production of "secondary" biomarkers in neighbouring cell types. Such secondary biomarkers are a source of candidate molecules that is absent when analysing the conditioned media from cell cultures. Co-cultures are likely to reflect this environment to a greater extent; however, the information associated with proximal biofluids such as pancreatic juice, saliva or nipple aspirate fluid takes in to account both primary and secondary biomarkers [78]. Although primary biomarkers are more disease specific, secondary biomarkers are likely to provide additional information, especially as part of a diagnostic panel. Biofluids are believed to harbour all available biomarkers, including protein fragments/peptides, modified and carrierbound proteins. Biofluids also exhibit different types of active extracellular proteins produced at various stages during the evolution of the disease. This situation is impossible to replicate in the lab; therefore, the emphasis is on identifying robust and uncomplicated candidate biomarkers in cell-culture-based investigations that have the possibility of being validated in clinical samples.

\section{Concluding remarks}

Presently, there is an immediate need for easily measurable biomarkers and associated cost-effective assays in the clinical setting. Highly sensitive and specific biomarkers that can aid in early detection, active monitoring of disease, monitoring of treatment response, identification of 
patients at different stages of the disease and identification of patients who will benefit from one treatment regime over another are needed. Such biomarkers will have an impact on disease diagnosis and management strategies [79]. It is also very likely that candidate biomarkers for early detection or monitoring could be useful as therapeutic targets.

It is clear that single biomarkers are unlikely to provide the sensitivity and specificity needed for screening the general population, assessing the effectiveness of treatment or monitoring relapse, given the substantial heterogeneity. In the future, panels of biomarkers are likely to be used in the clinic because of increased levels of overall accuracy. Members of these diagnostic panels are likely to be discovered in different biological samples using various experimental approaches. Therefore, the secretome including proteins released through various mechanisms such as classical secretion, non-classical secretory pathway and release through exosomes will play a central role in identifying such panel elements.

The authors have declared no conflict of interest.

\section{References}

[1] Yamori, T., Panel of human cancer cell lines provides valuable database for drug discovery and bioinformatics. Cancer Chemother. Pharmacol. 2003, 52, S74-S79.

[2] Dowling, P., Meleady, P., Dowd, A., Henry, M. et al., Proteomic analysis of isolated membrane fractions from superinvasive cancer cells. Biochim. Biophys. Acta 2007, 1774, 93-101.

[3] Xue, H., Lu, B., Lai, M., The cancer secretome: a reservoir of biomarkers. J. Transl. Med. 2008, 6, 52.

[4] Righetti, P. G., Castagna, A., Antonucci, F., Piubelli, C. et al., The proteome: anno Domini 2002. Clin. Chem. Lab. Med. 2003, 41, 425-438.

[5] Zhong, L., Roybal, J., Chaerkady, R., Zhang, W. et al., Identification of secreted proteins that mediate cell-cell interactions in an in vitro model of the lung cancer microenvironment. Cancer Res. 2008, 68, 7237-7245.

[6] Volmer, M. W., Stuhler, K., Zapatka, M., Schoneck, A. et al., Differential proteome analysis of conditioned media to detect Smad4 regulated secreted biomarkers in colon cancer. Proteomics 2005, 5, 2587-2601.

[7] Gronborg, M., Kristiansen, T. Z., Iwahori, A., Chang, R. et al., Biomarker discovery from pancreatic cancer secretome using a differential proteomic approach. Mol. Cell. Proteomics 2006, 5, 157-171.

[8] Weiss, J. V., Klein-Scory, S., Kubler, S., ReinacherSchick, A. et al., Soluble E-cadherin as a serum biomarker candidate: elevated levels in patients with late-stage colorectal carcinoma and FAP. Int. J. Cancer. 2010. Epub ahead of print.
[9] Klee, E. W., Sosa, C. P., Computational classification of classically secreted proteins. Drug Discov. Today 2007, 12, 234-240.

[10] Nickel, W., Pathways of unconventional protein secretion. Curr. Opin. Biotechnol. 2010, 5, 621-626.

[11] Andre, F., Schartz, N. E., Movassagh, M., Flament, C. et al., Malignant effusions and immunogenic tumour-derived exosomes. Lancet 2002, 360, 295-305.

[12] Zhou, M., Lucas, D. A., Chan, K. C., Issaq, H. J. et al., An investigation into the human serum "interactome". Electrophoresis 2004, 25, 1289-1298.

[13] Ayache, S., Panelli, M., Marincola, F. M., Stroncek, D. F., Effects of storage time and exogenous protease inhibitors on plasma protein levels. Am. J. Clin. Pathol. 2006, 126, 174-184.

[14] Gunawardana, C. G., Kuk, C., Smith, C. R., Batruch, I. et al., Comprehensive analysis of conditioned media from ovarian cancer cell lines identifies novel candidate markers of epithelial ovarian cancer. J. Proteome Res. 2009, 8, 4705-4713.

[15] Kulasingam, V., Diamandis, E. P., Tissue culture-based breast cancer biomarker discovery platform. Int. J. Cancer 2008, 123, 2007-2012.

[16] Martin, D. B, Gifford, D. R, Wright, M. E., Keller, A. et al., Quantitative proteomic analysis of proteins released by neoplastic prostate epithelium. Cancer Res. 2004, 64, 347-355.

[17] Wong, S. C., Chan, C. M., Ma, B. B., Lam, M. Y. et al., Advanced proteomic technologies for cancer biomarker discovery. Expert Rev. Proteomics 2009, 6, 123-134.

[18] Pellitteri-Hahn, M. C., Warren, M. C., Didier, D. N., Winkler, E. L. et al., Improved mass spectrometric proteomic profiling of the secretome of rat vascular endothelial cells. J. Proteome Res. 2006, 5, 2861-2864.

[19] Srisomsap, C., Sawangareetrakul, P., Subhasitanont, P., Chokchaichamnankit, D. et al., Proteomic studies of cholangiocarcinoma and hepatocellular carcinoma cell secretomes. J. Biomed. Biotechnol. 2010, 2010, 437143.

[20] Higa, L. M., Caruso, M. B., Canellas, F., Soares, M. R. et al., Secretome of HepG2 cells infected with dengue virus: implications for pathogenesis. Biochim. Biophys. Acta 2008, 1784, 1607-1616.

[21] Mbeunkui, F., Fodstad, O., Pannell, L. K., Secretory protein enrichment and analysis: an optimized approach applied on cancer cell lines using 2-D LC-MS/MS. J. Proteome Res. 2006, 5, 899-906.

[22] Weng, L. P., Wu, C. C., Hsu, B. L., Chi, L. M. et al., Secretome-based identification of Mac-2 binding protein as a potential oral cancer marker involved in cell growth and motility. J. Proteome Res. 2008, 7, 3765-3775.

[23] Zwickl, H., Traxler, E., Staettner, S., Parzefall, W. et al., A novel technique to specifically analyze the secretome of cells and tissues. Electrophoresis 2005, 26, 2779-2785.

[24] Gundacker, N. C., Haudek, V. J., Wimmer, H., Slany, A. et al., Cytoplasmic proteome and secretome profiles of differently stimulated human dendritic cells. J Proteome Res. 2009, 8, 2799-2811.

[25] Colzani, M., Waridel, P., Laurent, J., Faes, E. et al., Metabolic labeling and protein linearization technology allow the 
study of proteins secreted by cultured cells in serumcontaining media. J. Proteome Res. 2009, 8, 4779-4788.

[26] Jiang, L., He, L., Fountoulakis, M., Comparison of protein precipitation methods for sample preparation prior to proteomic analysis. J. Chromatogr. A 2004, 1023, 317-320.

[27] Chang, K. P., Wu, C. C., Chen, H. C., Chen, S. J. et al., Identification of candidate nasopharyngeal carcinoma serum biomarkers by cancer cell secretome and tissue transcriptome analysis: potential usage of cystatin A for predicting nodal stage and poor prognosis. Proteomics 2010, 10, 2644-2660.

[28] Slany, A., Haudek, V. J., Zwickl, H., Gundacker, N. C. et al., Cell characterization by proteome profiling applied to primary hepatocytes and hepatocyte cell lines Hep-G2 and Hep-3B. J. Proteome Res. 2010, 9, 6-21.

[29] Mathias, R. A., Wang, B., Ji, H., Kapp, E. A. et al., Secretome-based proteomic profiling of Ras-transformed MDCK cells reveals extracellular modulators of epithelialmesenchymal transition. J. Proteome Res. 2009, 8, 2827-2837.

[30] Currid, C. A., O'Connor, D. P., Chang, B. D., Gebus, C. et al., Proteomic analysis of factors released from p21-overexpressing tumour cells. Proteomics 2006, 6, 3739-3753.

[31] Baggetta, R., De Andrea, M., Gariano, G. R., Mondini, M. et al., The interferon-inducible gene IFI16 secretome of endothelial cells drives the early steps of the inflammatory response. Eur. J. Immunol. 2010, 40, 2182-2189.

[32] Chenau, J., Michelland, S., de Fraipont, F., Josserand, V. et al., The cell line secretome, a suitable tool for investigating proteins released in vivo by tumors: application to the study of p53-modulated proteins secreted in lung cancer cells. J. Proteome Res. 2009, 8, 4579-4591.

[33] Mauri, P., Scarpa, A., Nascimbeni, A. C., Benazzi, L. et al., Identification of proteins released by pancreatic cancer cells by multidimensional protein identification technology: a strategy for identification of novel cancer markers. FASEB J. 2005, 19, 1125-1127.

[34] Kashyap, M. K., Harsha, H. C., Renuse, S., Pawar, H. et al., SILAC-based quantitative proteomic approach to identify potential biomarkers from the esophageal squamous cell carcinoma secretome. Cancer Biol. Ther. 2010, 8, 796-810.

[35] Xu, B. J., Yan, W., Jovanovic, B., An, A. Q. et al., Quantitative analysis of the secretome of TGF-beta signalingdeficient mammary fibroblasts. Proteomics 201010 2458-2470.

[36] Gao, B. B., Stuart, L., Feener, E. P., Label-free quantitative analysis of one-dimensional PAGE LC/MS/MS proteome: application on angiotensin II-stimulated smooth muscle cells secretome. Mol. Cell. Proteomics 2008, 7, 2399-2409.

[37] Schiarea, S., Solinas, G., Allavena, P., Scigliuolo, G. M. et al., Secretome analysis of multiple pancreatic cancer cell lines reveals perturbations of key functional networks. $J$. Proteome Res. 2010, 9, 4376-4392.

[38] Taylor, C. R., Levenson, R. M., Quantification of immunohistochemistry - issues concerning methods, utility and semiquantitative assessment II. Histopathology 2006, 49, 411-424.
[39] Choi, S., Kim, J., Yea, K., Suh, P. G. et al., Targeted labelfree quantitative analysis of secretory proteins from adipocytes in response to oxidative stress. Anal. Biochem. 2010, 401, 196-202.

[40] Whiteaker, J. R., Zhao, L., Anderson, L., Paulovich, A. G., An automated and multiplexed method for high throughput peptide immunoaffinity enrichment and multiple reaction monitoring mass spectrometry-based quantification of protein biomarkers. Mol. Cell. Proteomics 2010, 9, 184-196.

[41] Zimmet, P., Alberti, K. G., Shaw, J., Global and societal implications of the diabetes epidemic. Nature 2001, 414, 782-787.

[42] Stumvoll, M., Goldstein, B. J., van Haeften, T. W., Type 2 diabetes: principles of pathogenesis and therapy. Lancet 2005, 365, 1333-1346.

[43] Mlinar, B., Marc, J., Janez, A., Pfeifer, M., Molecular mechanisms of insulin resistance and associated diseases. Clin. Chim. Acta 2007, 375, 20-35.

[44] Mohamadi, A., Cooke, D. W., Type 2 diabetes mellitus in children and adolescents. Adolesc. Med. State Art Rev. 2010, 21, 103-119.

[45] Rittig, K., Hieronimus, A., Thamer, C., Machann, J. et al., Reducing visceral adipose tissue mass is essential for improving endothelial function in type 2 diabetes prone individuals. Atherosclerosis. 2010, 2, 575-579.

[46] Breitling, R., Robust signaling networks of the adipose secretome. Trends Endocrinol. Metab. 2009, 20, 1-7.

[47] Zvonic, S., Lefevre, M., Kilroy, G., Floyd, Z. E. et al., Secretome of primary cultures of human adipose-derived stem cells: modulation of serpins by adipogenesis. Mol. Cell. Proteomics 2007, 6, 18-28.

[48] Zhou, H., Xiao, Y., Li, R., Hong, S. et al., Quantitative analysis of secretome from adipocytes regulated by insulin. Acta Biochim. Biophys. Sin. (Shanghai) 2009, 41, 910-921.

[49] Dowling, P., Shields, W., Rani, S., Meleady, P. et al., Proteomic analysis of conditioned media from glucose responsive and glucose non-responsive phenotypes reveals a panel of secreted proteins associated with beta cell dysfunction. Electrophoresis 2008, 29, 4141-4149.

[50] Song, G., Cui, Y., Zhong, N., Han, J., Proteomic characterisation of pancreatic islet beta-cells stimulated with pancreatic carcinoma cell conditioned medium. J. Clin. Pathol. 2009, 62, 802-807.

[51] Jemal, A., Siegel, R., Xu, J., Ward, E., Cancer statistics, 2010. CA Cancer J. Clin. 2010, 5, 277-300.

[52] Griffith, O. L., Chiu, C. G., Gown, A. M., Jones, S. J., Wiseman, S. M., Biomarker panel diagnosis of thyroid cancer: a critical review. Expert Rev. Anticancer Ther. 2008, 8, 1399-1413.

[53] Patz, E. F., Jr., Campa, M. J., Gottlin, E. B., Kusmartseva, I. et al., Panel of serum biomarkers for the diagnosis of lung cancer. J. Clin. Oncol. 2007, 25, 5578-5583.

[54] Matsumoto, T., Kawashima, Y., Nagashio, R., Kageyama, T. et al., A new possible lung cancer marker: VGF detection from the conditioned medium of pulmonary large cell 
neuroendocrine carcinoma-derived cells using secretome analysis. Int. J. Biol. Markers 2009, 24, 282-285.

[55] Arango, B. A., Castrellon, A. B., Perez, C. A., Raez, L. E., Santos, E. S., Nasopharyngeal carcinoma: alternative treatment options after disease progression. Expert Rev. Anticancer Ther. 2010, 10, 377-386.

[56] Chang, Y. H., Wu, C. C., Chang, K. P., Yu, J. S. et al., Cell secretome analysis using hollow fiber culture system leads to the discovery of CLIC1 protein as a novel plasma marker for nasopharyngeal carcinoma. J. Proteome Res. 2009, 8, 5465-5474.

[57] Wang, C. L., Wang, C. I., Liao, P. C., Chen, C. D. et al., Discovery of retinoblastoma-associated binding protein 46 as a novel prognostic marker for distant metastasis in nonsmall cell lung cancer by combined analysis of cancer cell secretome and pleural effusion proteome. J. Proteome Res. 2009, 8, 4428-4440.

[58] Mbeunkui, F., Metge, B. J., Shevde, L. A., Pannell, L. K., Identification of differentially secreted biomarkers using LC-MS/MS in isogenic cell lines representing a progression of breast cancer. J. Proteome Res. 2007, 6, 2993-3002.

[59] Xue, H., Lu, B., Zhang, J., Wu, M. et al., Identification of serum biomarkers for colorectal cancer metastasis using a differential secretome approach. J. Proteome Res. 2010, 9, 545-555.

[60] Sardana, G., Jung, K., Stephan, C., Diamandis, E. P., Proteomic analysis of conditioned media from the PC3, LNCaP, and 22Rv1 prostate cancer cell lines: discovery and validation of candidate prostate cancer biomarkers. J. Proteome Res. 2008, 7, 3329-3338.

[61] Kulasingam, V., Diamandis, E. P., Proteomics analysis of conditioned media from three breast cancer cell lines: a mine for biomarkers and therapeutic targets. Mol. Cell. Proteomics 2007, 6, 1997-2011.

[62] Lahat, G., Zhu, Q. S., Huang, K. L., Wang, S. et al., Vimentin is a novel anti-cancer therapeutic target; insights from in vitro and in vivo mice xenograft studies. PLoS One 2010, 5 , e10105.

[63] Hebert, L. E., Scherr, P. A., Bienias, J. L., Bennett, D. A., Evans, D. A., Alzheimer disease in the US population: prevalence estimates using the 2000 census. Arch. Neurol. 2003, 60, 1119-1122.

[64] Wancata, J., Musalek, M., Alexandrowicz, R., Krautgartner, M., Number of dementia sufferers in Europe between the years 2000 and 2050. Eur. Psychiatry 2003, 18, 306-313.

[65] Liu, B., Hong, J. S., Role of microglia in inflammationmediated neurodegenerative diseases: mechanisms and strategies for therapeutic intervention. J. Pharmacol. Exp. Ther. 2003, 304, 1-7.
[66] Agostinho, P., Cunha, R. A., Oliveira, C., Neuroinflammation, oxidative stress and the pathogenesis of Alzheimer's disease. Curr. Pharm. Des. 2010, 25, 2766-2778.

[67] Tansey, M. G., Goldberg, M. S., Neuroinflammation in Parkinson's disease: its role in neuronal death and implications for therapeutic intervention. Neurobiol. Dis. 2010, 37, 510-518.

[68] Suk, K., Combined analysis of the glia secretome and the CSF proteome: neuroinflammation and novel biomarkers. Expert Rev. Proteomics 2010, 7, 263-274.

[69] Kim, S., Ock, J., Kim, A. K., Lee, H. W. et al., Neurotoxicity of microglial cathepsin $D$ revealed by secretome analysis. $J$. Neurochem. 2007, 103, 2640-2650.

[70] Jeon, H., Lee, S., Lee, W. H., Suk, K., Analysis of glial secretome: the long pentraxin PTX3 modulates phagocytic activity of microglia. J. Neuroimmunol. 2010, 1-2, 63-72.

[71] Hur, J., Lee, P., Kim, M. J., Kim, Y., Cho, Y. W., Ischemiaactivated microglia induces neuronal injury via activation of gp91phox NADPH oxidase. Biochem. Biophys. Res. Commun. 2010, 391, 1526-1530.

[72] Marusyk, A., Polyak, K., Tumor heterogeneity: causes and consequences. Biochim. Biophys. Acta 2010, 1805, 105-117.

[73] Ween, M. P., Lokman, N. A., Hoffmann, P., Rodgers, R. J. et al., Transforming growth factor-beta-induced protein secreted by peritoneal cells increases the metastatic potential of ovarian cancer cells. Int. J. Cancer. 2010, Epub ahead of print.

[74] Wu, H. Y., Chang, Y. H., Chang, Y. C., Liao, P. C., Proteomics analysis of nasopharyngeal carcinoma cell secretome using a hollow fiber culture system and mass spectrometry. $J$. Proteome Res. 2009, 8, 380-389.

[75] Lipman, N. S., Jackson, L. R., Hollow fibre bioreactors: an alternative to murine ascites for small scale ( $<1$ gram) monoclonal antibody production. Res. Immunol. 1998, 149, 571-576.

[76] Cabrera, C. M., Cobo, F., Nieto, A., Cortes, J. L. et al., Identity tests: determination of cell line cross-contamination. Cytotechnology 2006, 51, 45-50.

[77] Buehring, G. C., Eby, E. A., Eby, M. J., Cell line crosscontamination: how aware are Mammalian cell culturists of the problem and how to monitor it? In Vitro Cell. Dev. Biol. Anim. 2004, 40, 211-215.

[78] Jesneck, J. L., Mukherjee, S., Yurkovetsky, Z., Clyde, M. et al., Do serum biomarkers really measure breast cancer? Biomed. Chromatogr. Cancer 2009, 9, 164.

[79] Foster, C. S., Cooper, C. S., Urgent need to develop independent biomarkers for functional, diagnostic and prognostic application in oncology research. Biomarkers Med. 2009, 3, 329-333. 